\title{
SMOOTH NUMBERS IN BEATTY SEQUENCES
}

\author{
ROGER BAKER
}

\begin{abstract}
Let $\theta$ be an irrational number of finite type and let $\psi \geq 0$. We consider numbers in the Beatty sequence of integer parts,

$$
\mathcal{B}(x)=\{\lfloor\theta n+\psi\rfloor: 1 \leq n \leq x\} .
$$

Let $C>3$. Writing $P(n)$ for the largest prime factor of $n$ and $|\ldots|$ for cardinality, we show that

$$
|\{n \in \mathcal{B}(x): P(n) \leq y\}|=\frac{1}{\theta} \Psi(\theta x, y)(1+o(1))
$$

as $x \rightarrow \infty$, uniformly for $y \geq(\log x)^{C}$. Here $\Psi(X, y)$ denotes the number of integers up to $X$ with $P(n) \leq y$. The range of $y$ extends that given by Akbal [1. The work of Harper [8] plays a key role in the proof.
\end{abstract}

\section{INTRODUCTION}

A positive integer $n$ is said to be $y$-smooth if $P(n)$, the largest prime factor of $n$, is at most $y$. We write $\mathfrak{S}(y)$ for the set of $y$-smooth numbers in $\mathbb{N}$ and

$$
\Psi(x, y)=|\mathfrak{S}(y) \cap[1, x]|,
$$

where $|\ldots|$ denotes cardinality.

Let $\theta>1$ be an irrational number and $\psi \in[0, \infty)$. Arithmetic properties of the Beatty sequence

$$
\mathcal{B}(x)=\{\lfloor\theta n+\psi\rfloor: 1 \leq n \leq x\}
$$

(where \lfloor\rfloor denotes integer part), have been studied in [1, 3, 4, 7], for example. One may conjecture that for $x$ large and $y=y(x)$ not too small, say $x \geq y \geq(\log x)^{C}$ where $C>1$, we have

$$
|\{n \in \mathcal{B}(x): P(n) \leq y\}|=\frac{1}{\theta} \Psi(\theta x, y) \quad(1+o(1))
$$

2020 Mathematics Subject Classification. Primary 11N25; secondary 11L03.

Key words and phrases. Beatty sequence, exponential sums over smooth numbers. 
where $o(1)$ denotes a quantity tending to 0 as $x$ tends to infinity. Banks and Shparlinski [4] obtained (1.1) (in a slightly different form) uniformly for

$$
\exp \left((\log x)^{2 / 3+\varepsilon}\right) \leq y \leq x
$$

(We write $\varepsilon$ for an arbitrary positive number.) Under the additional condition that $\theta$ is of finite type, Akbal [1] obtained (1.1) uniformly for

$$
\exp \left((\log \log x)^{5 / 3+\varepsilon}\right) \leq y \leq x .
$$

In the present paper we extend the range (1.2).

Theorem 1. Let $\theta>1$ be an irrational number of finite type and $\psi \geq 0$. Then (1.1) holds uniformly for

$$
(\log x)^{3+\varepsilon} \leq y \leq x
$$

We recall that an irrational number $\theta$ is said to be of finite type if ( $\mid \ldots . . \|$ denoting distance to the nearest integer) we have

$$
\|m \theta\| \geq \frac{c}{m^{\kappa}} \quad(m \in \mathbb{N})
$$

for some $c>0$ and $\kappa>0$. We note that if $\theta$ is of finite type, then so is $\theta^{-1}$.

Theorem 1 depends on an estimate for the exponential sum

$$
S(\theta)=\sum_{\substack{n \leq x \\ n \in \mathfrak{S}(y)}} e(n \theta)
$$

Akbal [1] uses the estimate of Foury and Tenenbaum [6]: for $3 \leq y \leq$ $\sqrt{x}, q \in \mathbb{N},(a, q)=1$, and $\delta \in \mathbb{R}$

$$
S\left(\frac{a}{q}+\delta\right) \ll x(1+|\delta x|) \log ^{3} x\left(\frac{y^{1 / 2}}{x^{1 / 4}}+\frac{1}{q^{1 / 2}}+\left(\frac{q y}{x}\right)^{1 / 2}\right) .
$$

This is unhelpful when, say, $y=(\log x)^{C}(C>1)$ since the trivial bound for $S(\theta)$ is $x^{1-1 / C+o(1)}$ (see e.g. [9]).

We use a procedure of Harper [8, Theorem 1] and to state his result we introduce some notation. For $2 \leq y \leq x$, let $u=\frac{\log y}{\log x}$ and let $\alpha=\alpha(x, y)$ be the solution of

$$
\sum_{p \leq y} \frac{\log p}{p^{\alpha}-1}=\log x
$$


For convenience, when $\theta=\frac{a}{q}+\delta$ as above, we write

$$
L=2(1+|\delta x|)
$$

and

$$
M=u^{3 / 2} \log u \log x(\log L)^{1 / 2}(\log q y)^{1 / 2} .
$$

In Theorem 1 of [8] it is shown that whenever

$$
q^{2} L^{2} y^{3} \leq x
$$

we have

$$
S\left(\frac{a}{q}+\delta\right) \ll \Psi(x, y)\left(q(1+|\delta x|)^{-\frac{1}{2}+\frac{3}{2}(1-\alpha(x, y))} M .\right.
$$

We cannot use this bound directly since (1.3) is too restrictive. We adapt Harper's argument to obtain

Theorem 2. Let $f$ be a completely multiplicative function, $|f(n)| \leq 1$ $(n \in \mathbb{N})$. Let

$$
S(f, \theta)=\sum_{\substack{n \leq x \\ n \in \mathfrak{S}(y)}} f(n) e(\theta n) .
$$

Let $q \in \mathbb{N},(a, q)=1$, and $\delta \in \mathbb{R}$. Then, with $\alpha=\alpha(x, y)$, we have

$S\left(f, \frac{a}{q}+\delta\right) \ll \Psi(x, y)\left\{\left(q(1+|\delta x|)^{-\frac{1}{2}+\frac{3}{2}(1-\alpha)} M+x^{\alpha / 2}\left(q L y^{3}\right)^{\frac{1}{2}} \sqrt{\log y \log q}\right\}\right.$.

To save space, we refer frequently to [8] in our proof of Theorem 2 in Section 2. Theorem 1 is deduced in a straightforward manner from Theorem 2 in Section 3.

The factor $f(n)$ in Theorem 2 is not needed elsewhere in the paper, but it requires no significant effort to include it.

I would like to thank Adam Harper for helpful comments concerning his proof of (1.5).

\section{Proof of Theorem 2.}

Lemma 1. Let $2 \leq y \leq x$ and $d \geq 1$. Then we have

$$
\Psi\left(\frac{x}{d}, y\right) \ll \frac{1}{d^{\alpha(x, y)}} \Psi(x, y) .
$$

Proof. See de la Bretèche and Tenenbaum [5, Théorème 2.4 (i)] 
We write $p(n)$ for the smallest prime factor of $n \in \mathbb{N}$. We begin the proof of Theorem 2 by noting that the result is trivial for $q L y^{3} \geq x^{\alpha}$. Suppose now that $q L y^{3}<x^{\alpha}$. Every $y$-smooth number in $\left[q L y^{2}, x\right]$ can be written uniquely in the form $m n$, where

$$
q L y<m \leq q L y^{2}, \frac{m}{P(m)} \leq q L y, m \in \mathfrak{S}(y)
$$

and

$$
\frac{q L y^{2}}{m} \leq n \leq \frac{x}{m}, p(n) \geq P(m), \quad n \in \mathfrak{S}(y) .
$$

(We take $m$ to consist of the product of the smallest prime factors of the number.) With $\theta=\frac{a}{q}+\delta$, we have

$$
\begin{aligned}
S(f, \theta) & =\sum_{\substack{q L y^{2} \leq n \leq x \\
n \in \mathfrak{S}(y)}} f(n) e(n \theta)+O\left(\Psi\left(q L y^{2}, y\right)\right) \\
& =U+O\left(\Psi\left(q L y^{2}, y\right)\right)
\end{aligned}
$$

where

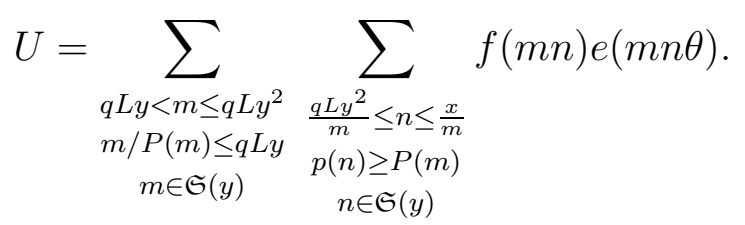

We now decompose $U$ as

$$
U=\sum_{0 \leq j \leq \log y} U_{j}
$$

where

$$
U_{j}=\sum_{p \leq y} \sum_{\substack{2^{j} q L y<m \leq q L y \min \left(2^{j+1}, p\right) \\ P(m)=p}} f(m) \sum_{\substack{\frac{q L y^{2}}{m} \leq n \leq \frac{x}{m} \\ p(n) \geq p \\ n \in \mathfrak{S}(y)}} f(n) e(m n \theta),
$$

noting that if $P(m)=p \leq y$, then $m$ is $y$-smooth, and the condition $\frac{m}{P(m)} \leq q L y$ can be written as $m \leq q L y p$. 
We apply the Cauchy-Schwarz inequality to $U_{j}$. Let $\sum_{m}$ denote

$$
\sum_{\substack{2^{j} q L y<m \leq q L y \min \left(2^{j+1}, p\right) . \\ P(m)=p}}
$$

We obtain

$$
\begin{aligned}
U_{j} & \leq \sqrt{\sum_{p \leq y} \sum_{m} 1} \sqrt{\sum_{2^{j} \leq p \leq y}} \sum_{\frac{2^{j} q L y}{p}<m^{\prime} \leq \frac{q L y}{p} \min \left(2^{j+1}, p\right)}\left|\sum_{\frac{q L y^{2}}{m^{\prime} p} \leq n \leq \frac{x}{m^{\prime} p}, p(n) \geq p, n \in \mathfrak{S}(y)} f(n) e\left(m^{\prime} p n \theta\right)\right|^{2} \\
& \ll \sqrt{\Psi\left(2^{j+1} q L y, y\right)} \sqrt{\sum_{\substack{2^{j} \leq p \leq y \\
n_{1}, n_{2} \leq \frac{x}{2^{j} q L y} \\
p\left(n_{1}\right), p\left(n_{2}\right) \geq p \\
n_{1}, n_{2} \in \mathfrak{S}(y)}} \min \left\{\frac{2^{j+1} q L y}{p}, \frac{1}{\left\|\left(n_{1}-n_{2}\right) p \theta\right\|}\right\}}
\end{aligned}
$$

For the last step, we open the square and sum the geometric progression over $m^{\prime}$. We may restrict the sum over primes to $p \geq 2^{j}$, since otherwise the sum over $m$ is empty. Our final bound here for $U_{j}$ exactly matches [8].

Let

$$
\mathfrak{T}_{j}(r):=\max _{1 \leq b \leq r-1} \sum_{\substack{n_{1}, n_{2} \leq \frac{x}{2^{j} q L y} \\ n_{1}, n_{2} \in \mathfrak{S}(y) \\ n_{1}-n_{2} \equiv b \bmod r}} 1 .
$$

Just as in [8], after distinguishing the cases $p \mid q$ and $p \nmid q$, we arrive at

$$
U_{j} \ll \sqrt{\Psi\left(2^{j+1} q L y, y\right)}\left(\sqrt{S}_{1}+\sqrt{S}_{2}\right),
$$

with

$$
\begin{aligned}
S_{1}=S_{1}(j):= & \sum_{\substack{2^{j} \leq p \leq y \\
p \nmid q}} \mathfrak{T}_{j}(q) \sum_{b=1}^{q-1} \min \left\{\frac{2^{j+1} q L y}{p}, \frac{q}{p}\right\} \\
& +\sum_{\substack{2^{j} \leq p \leq y \\
p \mid q}} \mathfrak{T}_{j}\left(\frac{q}{p}\right) \sum_{b=1}^{(q / p)-1} \min \left\{\frac{2^{j+1} q L y}{p}, \frac{q}{p b}\right\}
\end{aligned}
$$


and

$$
\begin{aligned}
S_{2}= & S_{2}(j):=\sum_{\substack{2^{j} \leq p \leq y \\
p \nmid q}} \frac{1}{p} \sum_{\substack{n_{1}, n_{2} \leq \frac{x}{2^{j} q L y} \\
n_{1}-n_{2} \equiv 0 \bmod q \\
n_{1}, n_{2} \in \mathfrak{S}(y)}} \min \left\{2^{j+1} q L y, \frac{1}{\left|\left(n_{1}-n_{2}\right) \delta\right|}\right\} \\
& \sum_{2^{j} \leq p \leq y} \frac{1}{p \mid q} \sum_{\substack{n_{1}, n_{2} \leq \frac{x}{2^{j} q L y} \\
n_{1}-n_{2} \equiv 0 \bmod q / p \\
n_{1}, n_{2} \in \mathfrak{S}(y)}} \min \left\{2^{j+1} q L y, \frac{1}{\left|\left(n_{1}-n_{2}\right) \delta\right|}\right\} .
\end{aligned}
$$

We now depart to an extent from the argument in [8]; we have extra terms in the upper bounds in Lemma 2 below which arise because we do not have the upper bound (1.4).

Lemma 2. Let $(\log x)^{1.1} \leq y \leq x^{1 / 3}, q \geq 1$, and $L=2(1+|\delta x|)$. Then for any $j, 0 \leq j \leq \frac{\log y}{\log 2}$, and any prime $p \mid q$, we have

$$
\mathfrak{T}_{j}(q) \ll \frac{\Psi\left(x / 2^{j} q L y, y\right)^{2}}{q} q^{1-\alpha(x, y)} \log x+y \Psi\left(\frac{x}{2^{j} q L y}, y\right)
$$

and

$$
\begin{aligned}
\mathfrak{T}_{j}\left(\frac{q}{p}\right) \ll \frac{\Psi\left(x / 2^{j} q L y, y\right)^{2}}{q / p}\left(\frac{q}{p}\right)^{1-\alpha(x, y)} & \log x \\
& +y \Psi\left(\frac{x}{2^{j} q L y}, y\right) .
\end{aligned}
$$

Under the same hypotheses, we have

$$
\begin{aligned}
& \sum_{\substack{n_{1}, n_{2} \leq \frac{x}{2^{j} q L y} \\
n_{1}-n_{2} \equiv 0 \bmod q \\
n_{1}, n_{2} \in \mathfrak{S}(y)}} \min \left\{2^{j+1} q L y, \frac{1}{\left|\left(n_{1}-n_{2}\right) \delta\right|}\right\} \\
& \ll 2^{j} y \Psi\left(\frac{x}{2^{j} q L y}, y\right)^{2}(q L)^{1-\alpha(x, y)} \log x \log L \\
& +2^{j} q L y^{2} \Psi\left(\frac{x}{2^{j} q L y}, y\right)
\end{aligned}
$$

and, for any prime $p \mid q$, 


$$
\begin{aligned}
& \sum_{\substack{n_{1}, n_{2} \leq \frac{x}{2^{j} q L y} \\
n_{1}-n_{2} \equiv 0 \bmod q / p \\
n_{1}, n_{2} \in \mathfrak{S}(y)}} \min \left\{2^{j+1} q L y, \frac{1}{\left|\left(n_{1}-n_{2}\right) \delta\right|}\right\} \\
& \ll p 2^{j} y \Psi\left(\frac{x}{2^{j} q L y}, y\right)^{2}\left(\frac{q L}{p}\right)^{1-\alpha(x, y)} \log x \log L \\
& +2^{j} q L y^{2} \Psi\left(\frac{x}{2^{j} q L y}, y\right) .
\end{aligned}
$$

Proof. We have

$$
\mathfrak{T}_{j}(q)=\max _{1 \leq b \leq q-1} \sum_{\substack{n_{1} \leq x / 2^{j} q L y \\ n_{1} \in \mathfrak{S}(y)}} \sum_{\substack{n_{2} \leq x / 2^{j} q L y \\ n_{2} \equiv n_{1}-b \bmod q \\ n_{2} \in \mathfrak{S}(y)}} 1,
$$

and just as in [8] the inner sum is

$$
\ll y+\frac{\Psi\left(x / 2^{j} q L y\right)}{q} q^{1-\alpha(x, y)} \log x .
$$

This leads to the bound (2.4) on summing over $n_{1}$. The bound (2.5) follows in exactly the same way.

To prove (2.6), (2.7) we distinguish two cases. If $|\delta| \leq 1 / x$, then $L=2(1+|\delta x|) \asymp 1$, and the bounds can be proved exactly as above on bounding $\min \left\{2^{j+1} q L y, \frac{1}{\left|\left(n_{1}-n_{2}\right) \delta\right|}\right\}$ by $2^{j+1} q L y \ll 2^{j} q y$.

Now suppose $|\delta|>1 / x$, so that $L \asymp|\delta x|$. We partition the sum in (2.6) dyadically. Let us use $\sum^{\dagger}$ to denote a sum over pairs of integers $n_{1}, n_{2} \leq \frac{x}{2^{j} q L y}$ that are $y$-smooth and satisfy $n_{1}-n_{2} \equiv 0 \bmod q$. Then we have

$$
\begin{aligned}
& \sum_{\left|n_{1}-n_{2}\right| \leq \frac{x}{2^{j} q L^{2} y}}^{\dagger} \min \left\{2^{j+1} q L y, \frac{1}{\left|\left(n_{1}-n_{2}\right) \delta\right|}\right\} \\
& \ll 2^{j} q L y \sum_{\substack{n_{1} \leq \frac{x}{2^{j} q L y} \\
n_{1} \in \mathfrak{S}(y)}} \sum_{\substack{\left|n_{2}-n_{1}\right| \leq \frac{x}{2^{j} q L^{2} y} \\
n_{2} \in \mathfrak{S}(y) \\
n_{2} \equiv n_{1} \bmod q}} 1 .
\end{aligned}
$$


Following [8], but as above not absorbing a term $y$, the last expression is

$$
\begin{aligned}
& \ll 2^{j} q L y \sum_{\substack{n_{1} \leq \frac{x}{2^{j} q L y} \\
n_{1} \in \mathfrak{S}(y)}}\left\{\frac{\Psi\left(x / 2^{j} q L y, y\right)}{q L}(q L)^{1-\alpha} \log x+y\right\} \\
& \ll 2^{j} y \Psi\left(x / 2^{j} q L y, y\right)^{2}(q L)^{1-\alpha} \log x+2^{j} q L y^{2} \Psi\left(x / 2^{j} q L y, y\right) .
\end{aligned}
$$

Similarly, for any $r, 0 \leq r \leq(\log L) / \log 2$, we have

$$
\begin{gathered}
\sum_{\frac{2^{r}}{2^{j} q L^{2} y}<\left|n_{1}-n_{2}\right| \leq \frac{2^{r+1} x}{2^{j} L^{2} y}}^{\dagger} \min \left\{2^{j+1} q L y, \frac{1}{\left|\left(n_{1}-n_{2}\right) \delta\right|}\right\} \\
\ll \frac{2^{j} q L y}{2^{r}} \sum_{\substack{n_{1} \leq \frac{x}{2^{j} q L y} \\
n_{1} \in \mathfrak{S}(y)}} \sum_{\substack{\left|n_{2}-n_{1}\right| \leq \frac{2}{}^{r+1} x \\
2^{j} L_{2} \in \mathfrak{S}(y) \\
n_{2} \equiv n_{1} \bmod q}} 1 \\
\ll \frac{2^{j} q L y}{2^{r}} \sum_{\substack{n_{1} \leq \frac{x}{2^{j} q L y} \\
n_{1} \in \mathfrak{S}(y)}}\left\{\Psi\left(\frac{x}{2^{j} q L y}, y\right)\left(\frac{2^{r}}{q L}\right)^{\alpha} \log x+y\right\} \\
\ll 2^{j} y \Psi\left(\frac{x}{2^{j} q L y}, y\right)^{2}\left(\frac{2^{r}}{q L}\right)^{\alpha} \log x+\frac{2^{j} q L y^{2}}{2^{r}} \Psi\left(\frac{x}{2^{j} q L y}, y\right) .
\end{gathered}
$$

The bound (2.6) follows on summing over $r$.

The bound (2.7) follows in exactly the same way; we lose a factor $p^{\alpha}$ in the first term on the right-hand side in (2.7) because of the weaker congruence condition. This completes the proof of Lemma 2 ,

We now assemble our bounds to prove Theorem 1. As noted in [8], for any $p \leq y$ we have

$$
\sum_{b=1}^{q-1} \min \left\{\frac{2^{j+1} q L y}{p}, \frac{q}{b}\right\} \ll q \log q
$$

and, for $p \mid q$,

$$
\sum_{b=1}^{(q / p)-1} \min \left\{\frac{2^{j+1} q L y}{p}, \frac{q}{p b}\right\} \ll \frac{q}{p} \log q
$$


Let

$$
A_{j}=\left(\sum_{2^{j} \leq p \leq y} \underset{p \nmid q}{p} \frac{1}{p}+\sum_{\substack{2^{j} \leq p \leq y \\ p \mid q}} 1\right) 2^{j} y \Psi\left(\frac{x}{2^{j} q L y}, y\right)(q L)^{1-\alpha} \log x \log L .
$$

We deduce from (2.1) $-(2.3)$, Lemma 2, and (2.8) $-(2.9)$ that

$$
S\left(f, \frac{a}{q}+\delta\right) \ll \Psi\left(q L y^{2}, y\right)+A+B_{1}+B_{2}
$$

where

$$
\begin{aligned}
A= & \sum_{0 \leq j \leq \frac{\log y}{\log 2}} \sqrt{\Psi\left(2^{j+1} q L y, y\right)} \sqrt{\frac{y}{\log y} \log q \Psi\left(\frac{x}{2^{j} q L y}, y\right)^{2} q^{1-\alpha} \log x} \\
& +\sum_{0 \leq j \leq \frac{\log y}{\log 2}} \sqrt{\Psi\left(2^{j+1} q L y, y\right)} \sqrt{A_{j}}, \\
B_{1}= & \sum_{0 \leq j \leq \frac{\log y}{\log 2}} \sqrt{\Psi\left(2^{j+1} q L y, y\right)} \sqrt{\sum_{2^{j} \leq p \leq y} q \log q \cdot y \Psi\left(\frac{x}{2^{j} q L y}, y\right)},
\end{aligned}
$$

and

$$
B_{2}=\sum_{0 \leq j \leq \frac{\log y}{\log 2}} \sqrt{\Psi\left(2^{j+1} q L y, y\right)} \sqrt{\frac{q L y^{3}}{\log y} \Psi\left(\frac{x}{2^{j} q y}, y\right)} .
$$

For the estimation of $A$ we can appeal to [8]:

$$
A \ll \Psi(x, y)(q L)^{-\frac{1}{2}+\frac{3}{2}(1-\alpha(x, y))} M .
$$

The $\Psi$ functions in $B_{1}$ and $B_{2}$ are estimated using Lemma 1 , Thus

$$
\begin{aligned}
\sqrt{\Psi\left(\frac{x}{2^{j} q L y}, y\right) \Psi\left(2^{j+1} q L y, y\right)} & \ll \Psi(x, y)\left(2^{j} q L y\right)^{-\alpha / 2}\left(\frac{x}{2^{j+1} q L y}\right)^{-\alpha / 2} \\
& \ll \Psi(x, y) x^{-\alpha / 2}
\end{aligned}
$$

leading to (a slightly wasteful) bound

$$
B_{1}+B_{2} \ll \Psi(x, y) \sqrt{y^{3} q L \log q \log y} x^{-\alpha / 2} .
$$


The remaining term to be estimated is $\Psi\left(q L y^{2}, y\right)$, and Lemma 1 gives

$$
\Psi\left(q L y^{2}, y\right) \ll\left(\frac{x}{q L y^{2}}\right)^{-\alpha} \Psi(x, y) .
$$

This term can be absorbed into the right-hand side of (2.12) since

$$
\left(q L y^{3}\right)^{\alpha-\frac{1}{2}} \ll\left(q L y^{3}\right)^{\alpha / 2} \ll x^{\alpha / 2} .
$$

Theorem 2 follows on combining (2.10)-(2.14).

\section{Proof of Theorem 2}

Lemma 3. Let $u_{1}, \ldots, u_{N} \in \mathbb{R}$. Then for any $J \in \mathbb{N}$ and any $\rho \leq \sigma \leq$ $\rho+1$, we have

$$
\begin{aligned}
||\left\{1 \leq n \leq N: u_{n}\right. & \in[\rho, \sigma] \bmod 1\}|-(\sigma-\rho) N| \\
& \leq \frac{N}{J+1}+3 \sum_{j=1}^{J} \frac{1}{j}\left|\sum_{n=1}^{N} e\left(j u_{n}\right)\right| .
\end{aligned}
$$

Proof. See e.g. 2], Theorem 2.1.

Proof of Theorem 1. In view of the result of [1] cited above, we may suppose that

$$
(\log x)^{3+\varepsilon} \leq y \leq \exp \left((\log \log x)^{2}\right)
$$

We note that $\lfloor\theta n+\psi\rfloor=m$ for some $m$ if and only if

$$
0<\left\{\frac{m+1-\psi}{\theta}\right\} \leq \frac{1}{\theta},
$$

so that, applying Lemma 3 .

$$
\begin{aligned}
\sum_{n \in \mathcal{B}(x, y)} 1 & =\sum_{\substack{m \leq \theta x \\
m \in \mathfrak{S}(y) \\
\text { (3.2) holds }}} 1+O(1) \\
& =\theta^{-1} \Psi(\theta x, y)+O\left(\frac{\Psi(\theta x, y)}{\log x}+\sum_{j=1}^{[\log x]} \frac{1}{j}\left|\sum_{\substack{m \leq \theta x \\
m \in \mathfrak{S}(y)}} e\left(\frac{j m}{\theta}\right)\right|\right)
\end{aligned}
$$


For our purposes, then, it suffices to show that

$$
\sum_{\substack{m \leq \theta x \\ m \in \mathfrak{S}(y)}} e\left(j \theta^{-1} m\right) \ll \Psi(\theta x, y)(\log x)^{-2}
$$

uniformly for $1 \leq j \leq \log x$. We deduce this from Theorem 2 .

By Dirichlet's theorem there is $q \in \mathbb{N}, 1 \leq q \leq x^{1 / 2}$, and $a \in \mathbb{N}$ with $(a, q)=1$ such that

$$
\delta:=j \theta^{-1}-\frac{a}{q} \in\left[-\frac{1}{q x^{1 / 2}}, \frac{1}{q x^{1 / 2}}\right] .
$$

Now

$$
\left|q j \theta^{-1}-a\right| \geq \frac{c_{1}}{(q j)^{\kappa}}
$$

for positive constants $c_{1}$ and $\kappa$, hence

$$
x^{-1 / 2} \geq q|\delta| \geq \frac{c_{1}}{(q j)^{\kappa}}
$$

giving

$$
q \gg x^{1 / 2 \kappa}(\log x)^{-1}
$$

We apply Theorem 2 with $j \theta^{-1}$ in place of $\theta$, and $\theta x$ in place of $x$. Note that

$$
q L=2 q(1+|\delta \theta x|) \ll x^{1 / 2}
$$

so that, in view of (3.1),

$$
q L y^{3} \ll x^{5 / 9} .
$$

Now, since $y \geq(\log x)^{3+\varepsilon}$, we have

$$
\alpha(\theta x, y) \geq \frac{2}{3}+\eta \quad(\eta=\eta(\varepsilon)>0) ;
$$

see [9], for example. Thus, abbreviating $\alpha(\theta x, y)$ to $\alpha$,

$$
q^{-\frac{1}{2}+\frac{3}{2}(1-\alpha)} \ll q^{-\eta} \ll x^{-\eta / 3 \kappa} .
$$

Now, with $M$ as in (1.3) with $\theta x$ in place of $x$,

$$
q^{-\frac{1}{2}+\frac{3}{2}(1-\alpha)} M \ll x^{-\eta / 4 \kappa} .
$$

Next, recalling (3.4),

$$
(\theta x)^{-\alpha / 2}\left(q L y^{3}\right)^{1 / 2} \sqrt{\log y \log q} \ll x^{-1 / 20} .
$$


Combining (3.5), (3.6) we obtain (3.3). This completes the proof of Theorem [1.

\section{REFERENCES}

[1] Y. Akbal, A note on values of Beatty sequences that are free of large prime factors, Colloq. Math. 160 (2020), 53-63.

[2] R.C. Baker, Diophantine Inequalities, Clarendon Press, Oxford, 1986.

[3] R.C. Baker and L. Zhao, Gaps between primes in Beatty sequences, Acta Arith. 172 (2016), 207-242.

[4] W.D. Banks and I.E. Shparlinski, Prime divisors in Beatty sequences, J. Number Theory 123 (2007), 413-425.

[5] R. de la Brétèche and G. Tenenbaum, Propriétés statistiques des entiers friables, Ramanujan J. 9 (2005), 139-202.

[6] E. Fouvry and G. Tenenbaum, Entiers sans grand facteur premier en progressions arithmetiques, Proc. London Math. Soc. 63 (1991), 449-494.

[7] G. Harman, Primes in Beatty sequences in short intervals, Mathematika 62 (2016), 572-586.

[8] A. Harper, Minor arcs, mean values and restriction theory for exponential sums over smooth numbers, Compos. Math. 152 (2016), 1121-1158.

[9] A. Hildebrand and G. Tenenbaum, Integers without large prime factors, $J$. Théor. Nombres Bordeaux 5 (1993), 411-484.

Department of Mathematics

Brigham Young University

Provo, UT 84602, U.S.A

Email address: baker@math. byu.edu 